\title{
UK-India Health Partnership to Benefit Both Countries
}

\author{
Mala Rao and Bhupinder Sandhu \\ Mala.rao@imperial.ac.uk
}

\section{ABSTRACT}

The current sweeping healthcare reforms in the UK and India present many challenges, but may also offer physicians of Indian origin from both countries a unique opportunity to join forces with other healthcare professionals and strengthen the health collaboration for the benefit of all. Through shared learning and experience of 'what works' and contributing to innovation, they can make a real difference to improving healthcare and reducing inequalities in both countries.

Key words NHS, partnership, health collaboration

Cite as: Rao, M., Sandhu, B. (2012) UK-India health partnership to benefit both countries. The Physician 1(1): 10-11 DOI: 10.38192/1.1.1.3

In India, the liberalisation of the economy and the consequent growth in prosperity over the past few decades has enhanced her status to that of a global power and there is an evident rise in national pride and confidence. But this image masks a huge rise in socioeconomic and health inequalities, which are being addressed by Government. The Report of the Steering Committee on Health for the 12th Five Year Plan (2012-2017) published in February 20121 by the Planning Commission of India highlighted that the Government's 'foremost commitment was towards evolving Universal Access to Essential Health Care and Medicines, so that disparities in access to health care, particularly those faced by the disadvantaged and underserved segments of the population would be corrected'. This is a welcome commitment in a country where more than $80 \%$ of healthcare expenditure is paid out of pocket. An increase in public health expenditure from less than 1\% of GDP to $2.5 \%$ of GDP is planned by 2017 , and priority is being given to the strengthening of primary care, which is recognised as an essential means to achieving affordable universal access to healthcare.

What can British Association of Physicians of Indian Origin (BAPIO) who have experience of working in the National Health Service (NHS) contribute to and gain from improving healthcare in India in the light of these current plans? A recently published comparative study of the health systems of 14 developed countries by Ingleby et al. 2 drew several conclusions about the NHS, including: that it outperformed other high income countries on many measures, despite spending much less than most of them; it enjoyed the highest levels of public confidence and satisfaction of all the countries studied; and that the positive 
assessment may be associated with care which is more accessible and better organised through higher levels of investment over past years. These high scores are also likely to be attributable, at least in part, to the high quality of primary care which has been the centrepiece of the NHS since it began. Indeed, most NHS care, including preventive care and the management of chronic disease, as well as first-contact acute care, is delivered in a community setting to a good standard with universal coverage by primary care practitioners. Many of the primary care practitioners are BAPIO who have considerable experience and potential therefore to share their learning with Indian physicians and support India's strategy to strengthen its primary care.

The NHS, on the other hand, is currently facing severe financial challenges. The Government is requiring an unprecedented level of efficiency improvements and is introducing radical reforms with several key features including the opening up of the market with the aim of creating a greater diversity of healthcare providers and using competition with the hope of driving up efficiency. In addition, the commissioning of care is being handed to GP consortia, with the intention that commissioning is to be clinician-led.

In India, the healthcare landscape is one of public and private sector organisations co-existing with one another, and increasingly, working together as a result of innovative public-private partnership initiatives intended to address both public sector inefficiencies and private sector behaviours motivated by profit rather than ethics. Clinical leadership in the provision, management and commissioning of healthcare is de rigeur, and is offered as an explanation for the ability of Indian doctors, especially in the most admired institutions, to 'do more for less', and demonstrate high levels of innovation and entrepreneurship. The BAPIO working in the NHS can learn much from the experience of these Indian doctors. There is thus significant scope for mutual learning about what works, and perhaps more importantly, what doesn't work, in terms of healthcare commissioning, driving efficiency through competition, and private sector involvement, as well as providing ethical clinical leadership in innovative service provision and teaching and research.

Worldwide, it is estimated that there are 1.2 million doctors of Indian origin serving in a vast number of countries. India alone has 800,000 doctors. In the UK, the NHS has a higher representation of ethnic minority doctors in its medical workforce than in the general population. It employs over 40,000 BPIOs. It is estimated that there may be around 15,000 BPIOs in training who are interested in opportunities to work in India.

The UK Global Health Strategy 2008-2013 3 highlighted India among the priority countries for collaboration. It aimed to promote the best in British healthcare, to make an effective contribution to health in other countries and to utilise learning through partnerships to improve healthcare in the NHS. The potential for BPIOs, with their understanding of the language, culture and social conditions in the UK as well as India, puts them in a unique position to lead these partnerships and help strengthen the UK's health partnerships with India. The newly-launched Physician provides a timely opportunity for BPIOs to catalyse debate and discussion on these issues by sharing of information and dissemination of research evidence among all healthcare professionals committed to improving healthcare in India and the UK. 


\section{References}

1. Planning Commission of India, Health Division. Report of the Steering Committee on Health for the 12th Five Year Plan. February 2012.

http://planningcommission.nic.in/aboutus/committee/strgrp12/str_health0203.pdf

2. Ingleby D, McKee M, Mladovsky $P$, Rechel B. How the NHS measures up to other health systems. BMJ 2012; 344:e1079 doi

3. Department of Health for England. Health is Global: A UK Government strategy 2008-2013. London.http://www.dh.gov.uk/en/Publicationsandstatistics/Publications/PublicationsPolicyAnd Guidance /DH_088702 